\title{
Prognostic genes of melanoma identified by weighted gene co-expression network analysis and drug repositioning using a network-based method
}

\author{
LU WANG $^{1 *}$, CHUAN-YUAN WEI $^{1 *}$, YUAN-YUAN XU $^{2 *}$, XIN-YI DENG ${ }^{1}$, QIANG WANG ${ }^{1}$, \\ JIANG-HUI YING ${ }^{1}$, SI-MIN ZHANG ${ }^{1}$, XIN YUAN ${ }^{1}$, TIAN-FAN XUAN ${ }^{1}$, YU-YAN PAN ${ }^{1}$ and JIAN-YING GU ${ }^{1}$ \\ ${ }^{1}$ Department of Plastic Surgery, Zhongshan Hospital, Fudan University, Shanghai 200032; \\ ${ }^{2}$ Department of Surgery, The First Hospital of Shanxi Medical University, Taiyuan, Shanxi 030001, P.R. China
}

Received February 12, 2019; Accepted August 21, 2019

DOI: $10.3892 / 01.2019 .10961$

\begin{abstract}
Melanoma is one of the most malignant types of skin cancer. However, the efficacy and utility of available drug therapies for melanoma are limited. The objective of the present study was to identify potential genes associated with melanoma progression and to explore approved therapeutic drugs that target these genes. Weighted gene co-expression network analysis was used to construct a gene co-expression network, explore the associations between genes and clinical characteristics and identify potential biomarkers. Gene expression profiles of the GSE65904 dataset were obtained from the Gene Expression Omnibus database. RNA-sequencing data and clinical information associated with melanoma obtained from The Cancer Genome Atlas were used for biomarker validation. A total of 15 modules were identified through average linkage hierarchical clustering. In the two significant modules, three network hub genes associated with melanoma prognosis were identified: C-X-C motif chemokine receptor 4 (CXCR4), interleukin 7 receptor (IL7R) and phosphatidylinositol-4,5-bisphosphate 3-kinase catalytic subunit $\gamma$ (PIK3CG). The receiver operating
\end{abstract}

Correspondence to: Professor Jian-Ying Gu, Department of Plastic Surgery, Zhongshan Hospital, Fudan University, 180 Fenglin Road, Shanghai 200032, P.R. China

E-mail: zhongshanhospital@163.com

*Contributed equally

Abbreviations: WGCNA, weighted gene co-expression network analysis; GEO, Gene Expression Omnibus; ROC, receiver operating characteristic; GO, Gene Ontology; KEGG, Kyoto Encyclopedia of Genes and Genomes; BP, biological process; CC, cellular component; MF, molecular function; MEs, module eigengenes; GS, gene significance; MM, module membership; PPI, protein-protein interaction; TKIs, tyrosine kinase inhibitors; BTK, Burton's tyrosine kinase; VEGFR, vascular endothelial growth factor receptor

Key words: melanoma, prognosis, drug repositioning characteristic curve indicated that the mRNA levels of these genes exhibited excellent prognostic efficiency for primary and metastatic tumor tissues. In addition, the proximity between candidate genes associated with melanoma progression and drug targets obtained from DrugBank was calculated in the protein interaction network, and the top 15 drugs that may be suitable for treating melanoma were identified. In summary, co-expression network analysis led to the selection of CXCR4, IL7R and PIK3CG for further basic and clinical research on melanoma. Utilizing a network-based method, 15 drugs that exhibited potential for the treatment of melanoma were identified.

\section{Introduction}

Melanoma is a tumor that originates in melanocytes of the skin or other parts of the body (1). The main function of melanocytes is to produce melanin via melanogenesis, a multistep biochemical process regulated by L-tyrosine, L-DOPA and other hormones $(2,3)$. Melanogenesis leads to the upregulation of hypoxia-inducible factor 1 , which modulates the cellular metabolism of melanoma (4). A previous study has demonstrated that pigmentation level is associated with the overall and disease-free survival time of patients with stage III and IV melanoma (5). In the United States, $>91,000$ individuals were diagnosed with cutaneous melanoma in 2018 , and $>9,000$ patients succumbed to the disease in the same period (6). Since melanoma tends to spread lymphogenously and hematogenously, patients with inoperable metastatic melanoma exhibit median survival times between 8 and 12 months (7). Therefore, melanoma poses a serious threat to life.

Gene mutations in melanoma may activate multiple signaling pathways that regulate proliferation, epithelial-mesenchymal transition, invasion and metastasis in an abnormal manner (8). For example, BRAF mutations, predominantly V600E, occur in 40-50\% of all melanomas, whereas NRAS proto-oncogene, GTPase and neurofibromin 1 mutations occur in 20 and $15 \%$ of melanomas, respectively (9). Targeted therapy and immunotherapy have been demonstrated to be effective treatment methods $(10,11)$. $\mathrm{BRAF/mitogen-activated} \mathrm{protein} \mathrm{kinase} \mathrm{kinase} \mathrm{inhibitors,} \mathrm{as}$ 
well as antibodies against cytotoxic T-lymphocyte-associated protein 4 and programmed cell death protein 1 have been used for treatment of metastatic melanoma, with patient response rates ranging between 20 and $70 \%$ (12). Although these breakthrough treatments have prolonged progression-free survival to a certain extent, drug resistance still limits their effectiveness (13). For example, immune-based therapy is subject to limitations, such as the prevention of the generation of an immunosuppressive environment (14). Therefore, there remains a need for novel markers of prognosis and novel therapeutic drugs for melanoma.

Weighted gene co-expression network analysis (WGCNA) is widely used to analyze genetic expression data, locate modules of highly correlated genes and identify potential biomarkers, as well as therapeutic targets. Thus, the present study aimed to utilize WGCNA to identify novel biomarkers associated with melanoma prognosis. Additionally, the present study aimed to determine the proximity between disease-associated proteins and drug targets in the human protein-protein interactome in order to identify potential drugs for the treatment of melanoma.

\section{Materials and methods}

Data processing. Melanoma transcriptome dataset GSE65904 (15) was downloaded from the Gene Expression Omnibus (GEO) database (https://www.ncbi.nlm.nih.gov/geo). GSE65904 comprised 214 samples from patients with melanoma, no non-tumor tissue samples or healthy subjects were included. Illumina HumanHT-12V4.0 expression beadchip was used as the sequencing platform. Clinical information of patients, including sex, age, tumor stage, distant metastasis and survival state, was collected. The GEO query package in R v2.52.0 (https:/git.bioconductor.org/packages/GEOquery) was used to process the data. If the expression of a gene was not significant compared with the background value (standard probe) in $>25 \%$ of all samples $(\mathrm{P}>0.05)$, the probe was removed from further analysis. A total of 10,566 genes were obtained.

Weighted co-expression network construction. The top $50 \%$ most differentially expressed genes (5,283 genes) were selected for WGCNA analysis following analysis of variance using R 3.3.2 (https://www.r-project.org/) (16). These genes were used for screening and cluster analysis of all samples, as well as to identify outliers, following which one patient was removed from the study (Fig. 1). The gene expression data of the patients was used to construct the co-expression network, and the WGCNA algorithm was utilized for analysis (16). To ensure that the nodes of the constructed co-expression network conformed to the power rate distribution, appropriately soft threshold was selected $(\beta=3)$, which enabled the deletion of low mutual correlation relationships. The distribution of network nodes conformed to the power rate distribution at $\beta=3$. Further investigation of the distribution of node degrees in the co-expression network revealed that the degree of nodes conformed to the power law distribution. This indicated that the constructed co-expression network was a scale-free network, conforming to the characteristics of common biological networks. The average linkage hierarchical clustering method (17) was used to cluster all genes.
Identification of clinically significant modules. To obtain the gene modules that were associated with clinical phenotypes, the correlation between modules and clinical phenotypes was determined. Module eigengenes (MEs) were considered as characteristics of all genes in a certain module. The association between MEs and clinical characteristics was analyzed to determine a clinically significant module for further use.

Gene Ontology (GO) and pathway enrichment analysis. The ClusterProfiler package (https://github. com/GuangchuangYu/clusterProfiler) in R v3.12.0 was used to determine the functions of the enriched genes from the two modules in Fig. 3 (black and turquoise modules) in GO (18) and the Kyoto Encyclopedia of Genes and Genomes (KEGG) (19) pathway analysis, respectively. Genes in the clinically significant module were categorized into three functional groups: Biological process (BP), cellular component (CC) and molecular function (MF).

Identification and validation of hub genes. To identify genes associated with melanoma prognosis, the association between each gene and clinical characteristics was evaluated, as well as the association between each gene and core modules, such as module membership (MM) and gene significance (GS). $\mathrm{MM}$ is defined by the correlation between the gene expression profile and MEs, whereas GS is defined by the association between a gene and external traits. Genes with $|\mathrm{MM}+\mathrm{GS}|=5 \%$ in the aforementioned modules (black and turquoise modules) in Fig. 3 were selected as potentially prognostic genes; all other genes were removed. To further analyze the association between these genes, the remaining candidate genes were input into STRING (https://string-db.org/) to construct a protein-protein interaction (PPI) network using Cytoscape v3.2 (20).

To verify whether the identified genes were associated with tumor progression and prognosis, the association between each gene and survival was determined using the R survival package v2.41-3 (https://cran.r-project. org/web/packages/survminer/index.html). Clinical and RNA-sequencing data from 417 patients with melanoma were downloaded from The Cancer Genome Atlas (TCGA) database (https://cancergenome.nih.gov/) using the TCGA biolinks package in $\mathrm{R}$ v2.12.3 (https://git.bioconductor. org/packages/TCGAbiolinks). Overall survival was analyzed using the log-rank test. In addition, the ggpubr package v0.2.1 (http://cran.r-project.org/web/packages/ggpubr/index. html) was used to demonstrate the mRNA expression of hub genes in primary and metastatic tumor, and the two groups were compared by Student's t-test. Receiver operating characteristic (ROC) curve and area under the curve (AUC) values were obtained using the pROC package v1.15.0 (http://cran.r-project. org/web/packages/ROCR) to evaluate the efficiency of the genes in distinguishing metastatic and non-metastatic tumors.

Screening candidates for treatment. Drug-target information of Food and Drug Administration (FDA)-approved drugs was obtained from DrugBank (https://www.drugbank.ca/). The exclusion of drugs that had no known targets in the interactome resulted in a total of 1,269 unique drugs and 1,185 targets selected for further analysis. Notably, only pharmacological 


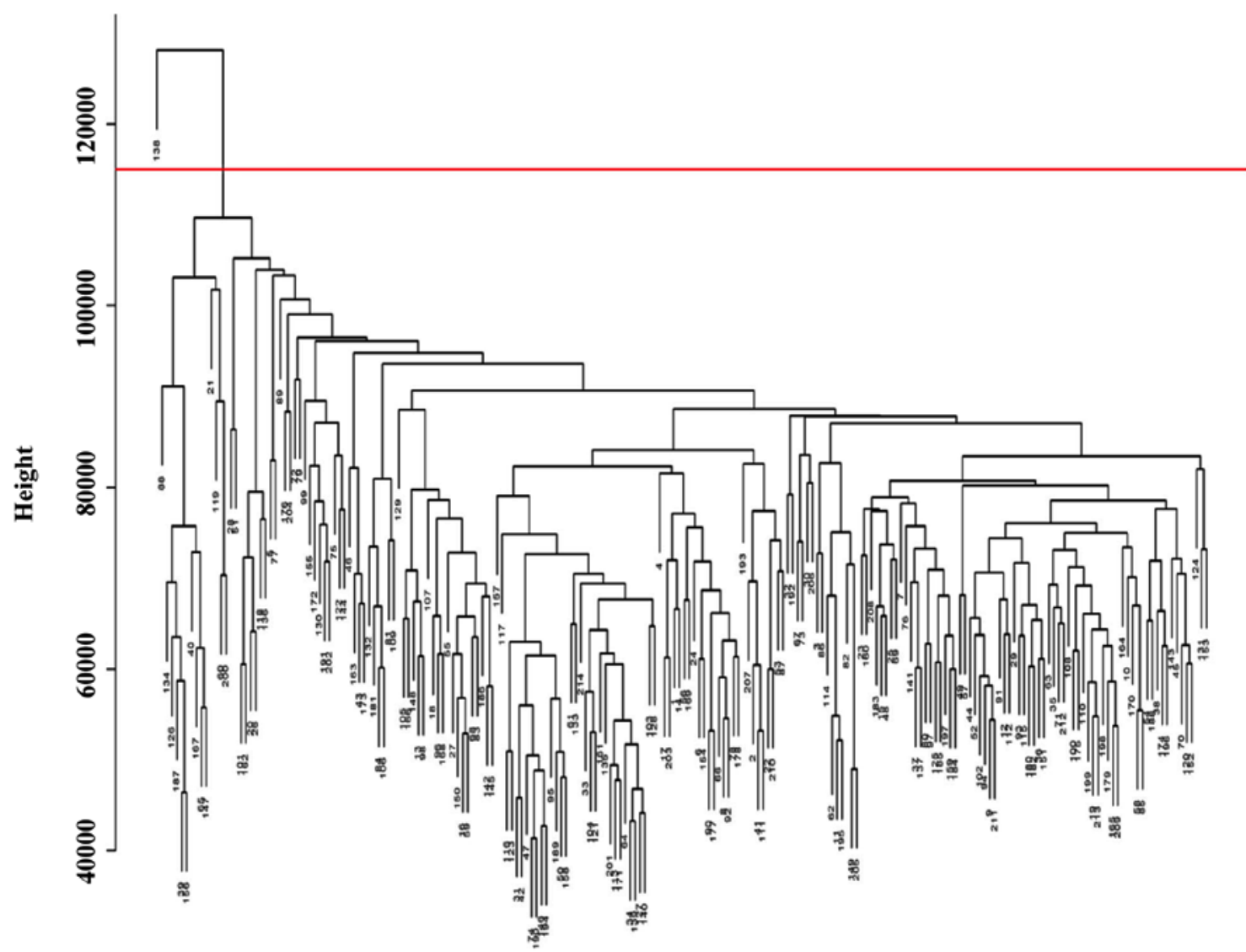

Figure 1. Cluster dendrogram of 214 melanoma samples. The GSE65904 dataset was used. The red line indicates the outlier to rule out biased samples. The black lines represent each sample in the dataset, and the numbers represent corresponding GSM of the patient.

targets ('Targets' section in DrugBank), excluding enzymes, carriers and transporters typically shared among different drugs, were considered. The protein interaction information was obtained from a previously published study, which contained data from 15 databases (21). Among these, 15,969 nodes and 217,160 mutual relationships were identified in the PPI networks. The prognostic genes of melanoma were mapped to the PPI network. The Igraph package v1.2.4.1 (https://igraph. org/) was used to estimate the shortest distance between each target and a particular prognostic gene for each FDA-approved drug (21). Standardization-based approximation indicated that lower values were associated with an increased likelihood that the drug may act on melanoma and prevent its progression.

\section{Results}

Weighted co-expression network construction and key module identification. Following a cluster analysis of all samples, one sample in GSE65904 was removed from subsequent analysis due to bias (Fig. 1; Table I). To ensure a scale-free network, it must satisfy $\mathrm{R}^{2}>0.8$ (Fig. 2A), and the mean connectivity should be conserved as much as possible (Fig. 2B). Furthermore, the degree distribution of nodes in the co-expression network was investigated further and the degree of nodes conforms to power law distribution (Fig. 2C and D). The WGCNA package in $\mathrm{R}$ was used to place genes with similar expression patterns into modules through average linkage clustering; a total of 15 modules were identified (Fig. 3A). The black module exhibited the strongest association with tumor metastasis-free survival and disease-specific death survival (Fig. 3), whereas the turquoise module exhibited the strongest association with tumor stage. Therefore, these two modules were considered to be clinically significant and were selected for further analysis.

$G O$ and KEGG pathway enrichment analysis. The genes in the clinically significant modules were categorized into functional groups: BP, CC and MF. The genes in the black module were mainly enriched in 'antigen processing and presentation', 'antigen processing and presentation of peptide antigen' and 'antigen processing and presentation of exogenous peptide antigen' in the BP group, 'endocytic vesicle membrane', 'Golgi-associated vesicle' and 'COPII-coated ER to Golgi transport vesicle' in the CC group, and 'amide binding', 'peptide binding' and 'antigen binding' in the MF group (Fig. 4A). The results of the KEGG pathway analysis demonstrated that genes in the black module were mainly involved in 'antigen processing and presentation', 'viral myocarditis', 'cell adhesion molecules cams' and 'allograft rejection', among others (Fig. 5A).

The genes in the turquoise module were mainly enriched in 'leukocyte differentiation', 'T cell activation' and 'regulation of lymphocyte activation' in the BP group, 'cell leading edge', 'lamellipodium' and 'cytoplasmic side of plasma membrane' in the CC group and 'nucleoside-triphosphatase regulator activity', 'GTPase regulator activity' and 'phospholipid binding' in the MF group (Fig. 4B). The results of 
Table I. Summary of number and corresponding GSM in GSE65904.

\begin{tabular}{|c|c|c|c|}
\hline & \\
\hline & & Number & GSM \\
\hline Number & GSM & & CCM1608644 \\
\hline 1 & GSM1608593 & 53 & GSM1608645 \\
\hline 2 & GSM1608594 & 54 & GSM1608646 \\
\hline 3 & GSM1608595 & 55 & GSM1608647 \\
\hline 4 & GSM1608596 & 56 & GSM1608648 \\
\hline 5 & GSM1608597 & 57 & GSM1608649 \\
\hline 6 & GSM1608598 & 58 & GSM1608650 \\
\hline 7 & GSM1608599 & 59 & GSM1608651 \\
\hline 8 & GSM1608600 & 60 & GSM1608652 \\
\hline 9 & GSM1608601 & 61 & GSM1608653 \\
\hline 10 & GSM1608602 & 62 & GSM1608654 \\
\hline 11 & GSM1608603 & 63 & GSM1608655 \\
\hline 12 & GSM1608604 & 64 & GSM1608656 \\
\hline 13 & GSM1608605 & 65 & GSM1608657 \\
\hline 14 & GSM1608606 & 66 & GSM1608658 \\
\hline 15 & GSM1608607 & 67 & GSM1608659 \\
\hline 16 & GSM1608608 & 68 & GSM1608660 \\
\hline 17 & GSM1608609 & 69 & GSM1608661 \\
\hline 18 & GSM1608610 & 70 & GSM1608662 \\
\hline 19 & GSM1608611 & 71 & GSM1608663 \\
\hline 20 & GSM1608612 & 72 & GSM1608664 \\
\hline 21 & GSM1608613 & 73 & GSM1608665 \\
\hline 22 & GSM1608614 & 74 & GSM1608666 \\
\hline 23 & GSM1608615 & 75 & GSM1608667 \\
\hline 24 & GSM1608616 & 76 & GSM1608668 \\
\hline 25 & GSM1608617 & 77 & GSM1608669 \\
\hline 26 & GSM1608618 & 78 & GSM1608670 \\
\hline 27 & GSM1608619 & 79 & GSM1608671 \\
\hline 28 & GSM1608620 & 80 & GSM1608672 \\
\hline 29 & GSM1608621 & 81 & GSM1608673 \\
\hline 30 & GSM1608622 & 82 & GSM1608674 \\
\hline 31 & GSM1608623 & 83 & GSM1608675 \\
\hline 32 & GSM1608624 & 84 & GSM1608676 \\
\hline 33 & GSM1608625 & 85 & GSM1608677 \\
\hline 34 & GSM1608626 & 86 & GSM1608678 \\
\hline 35 & GSM1608627 & 87 & GSM1608679 \\
\hline 36 & GSM1608628 & 88 & GSM1608680 \\
\hline 37 & GSM1608629 & 89 & GSM1608681 \\
\hline 38 & GSM1608630 & 90 & GSM1608682 \\
\hline 39 & GSM1608631 & 91 & GSM1608683 \\
\hline 40 & GSM1608632 & 92 & GSM1608684 \\
\hline 41 & GSM1608633 & 93 & GSM1608685 \\
\hline 42 & GSM1608634 & 94 & GSM1608686 \\
\hline 43 & GSM1608635 & 95 & GSM1608687 \\
\hline 44 & GSM1608636 & 96 & GSM1608688 \\
\hline 45 & GSM1608637 & 97 & GSM1608689 \\
\hline 46 & GSM1608638 & 98 & GSM1608690 \\
\hline 47 & GSM1608639 & 99 & GSM1608691 \\
\hline 48 & GSM1608640 & 100 & GSM1608692 \\
\hline 49 & GSM1608641 & 101 & GSM1608693 \\
\hline 50 & GSM1608642 & 102 & GSM1608694 \\
\hline 51 & GSM1608643 & 103 & GSM1608695 \\
\hline
\end{tabular}

Table I. Continued. 
Table I. Continued.

\begin{tabular}{|c|c|c|c|}
\hline Number & GSM & Number & GSM \\
\hline 104 & GSM1608696 & 156 & GSM1608748 \\
\hline 105 & GSM1608697 & 157 & GSM1608749 \\
\hline 106 & GSM1608698 & 158 & GSM1608750 \\
\hline 107 & GSM1608699 & 159 & GSM1608751 \\
\hline 108 & GSM1608700 & 160 & GSM1608752 \\
\hline 109 & GSM1608701 & 161 & GSM1608753 \\
\hline 110 & GSM1608702 & 162 & GSM1608754 \\
\hline 111 & GSM1608703 & 163 & GSM1608755 \\
\hline 112 & GSM1608704 & 164 & GSM1608756 \\
\hline 113 & GSM1608705 & 165 & GSM1608757 \\
\hline 114 & GSM1608706 & 166 & GSM1608758 \\
\hline 115 & GSM1608707 & 167 & GSM1608759 \\
\hline 116 & GSM1608708 & 168 & GSM1608760 \\
\hline 117 & GSM1608709 & 169 & GSM1608761 \\
\hline 118 & GSM1608710 & 170 & GSM1608762 \\
\hline 119 & GSM1608711 & 171 & GSM1608763 \\
\hline 120 & GSM1608712 & 172 & GSM1608764 \\
\hline 121 & GSM1608713 & 173 & GSM1608765 \\
\hline 122 & GSM1608714 & 174 & GSM1608766 \\
\hline 123 & GSM1608715 & 175 & GSM1608767 \\
\hline 124 & GSM1608716 & 176 & GSM1608768 \\
\hline 125 & GSM1608717 & 177 & GSM1608769 \\
\hline 126 & GSM1608718 & 178 & GSM1608770 \\
\hline 127 & GSM1608719 & 179 & GSM1608771 \\
\hline 128 & GSM1608720 & 180 & GSM1608772 \\
\hline 129 & GSM1608721 & 181 & GSM1608773 \\
\hline 130 & GSM1608722 & 182 & GSM1608774 \\
\hline 131 & GSM1608723 & 183 & GSM1608775 \\
\hline 132 & GSM1608724 & 184 & GSM1608776 \\
\hline 133 & GSM1608725 & 185 & GSM1608777 \\
\hline 134 & GSM1608726 & 186 & GSM1608778 \\
\hline 135 & GSM1608727 & 187 & GSM1608779 \\
\hline 136 & GSM1608728 & 188 & GSM1608780 \\
\hline 137 & GSM1608729 & 189 & GSM1608781 \\
\hline 138 & GSM1608730 & 190 & GSM1608782 \\
\hline 139 & GSM1608731 & 191 & GSM1608783 \\
\hline 140 & GSM1608732 & 192 & GSM1608784 \\
\hline 141 & GSM1608733 & 193 & GSM1608785 \\
\hline 142 & GSM1608734 & 194 & GSM1608786 \\
\hline 143 & GSM1608735 & 195 & GSM1608787 \\
\hline 144 & GSM1608736 & 196 & GSM1608788 \\
\hline 145 & GSM1608737 & 197 & GSM1608789 \\
\hline 146 & GSM1608738 & 198 & GSM1608790 \\
\hline 147 & GSM1608739 & 199 & GSM1608791 \\
\hline 148 & GSM1608740 & 200 & GSM1608792 \\
\hline 149 & GSM1608741 & 201 & GSM1608793 \\
\hline 150 & GSM1608742 & 202 & GSM1608794 \\
\hline 151 & GSM1608743 & 203 & GSM1608795 \\
\hline 152 & GSM1608744 & 204 & GSM1608796 \\
\hline 153 & GSM1608745 & 205 & GSM1608797 \\
\hline 154 & GSM1608746 & 206 & GSM1608798 \\
\hline 155 & GSM1608747 & 207 & GSM1608799 \\
\hline
\end{tabular}

Table I. Continued. 
Table I. Continued.

\begin{tabular}{lc}
\hline Number & GSM \\
\hline 208 & GSM1608800 \\
209 & GSM1608801 \\
210 & GSM1608802 \\
211 & GSM1608803 \\
212 & GSM1608804 \\
213 & GSM1608805 \\
214 & GSM1608806
\end{tabular}

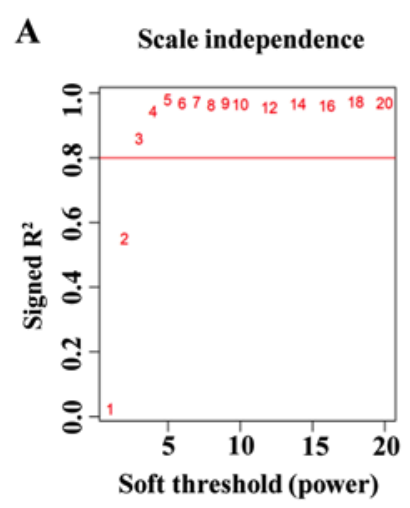

C

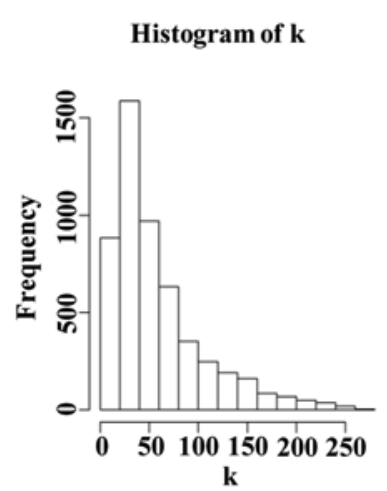

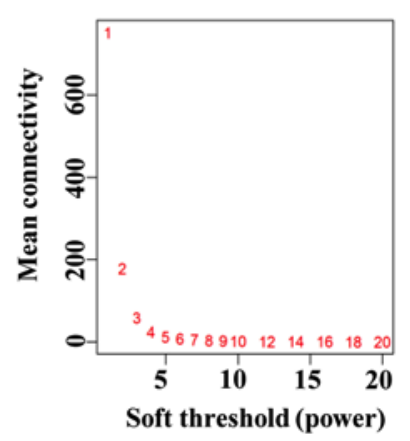

D

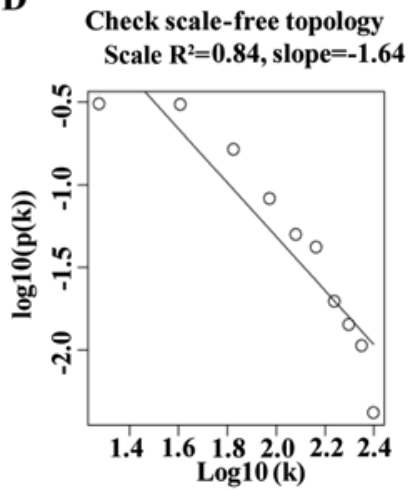

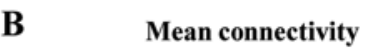

Figure 2. Determination of soft-thresholding power in weighted gene co-expression network analysis. (A) Scale-free fit index of various soft-thresholding powers. (B) Mean connectivity of various soft-thresholding powers. (C) Histogram of connectivity distribution at $\beta=3$. (D) Scale-free topology at $\beta=3$. $\beta$, soft thresholding power; $\mathrm{k}$, connectivity.

the KEGG pathway analysis demonstrated that the genes in the turquoise module were mainly involved in 'chemokine signaling pathway', 'B cell receptor signaling pathway' and ' $\mathrm{T}$ cell receptor signaling pathway', among others (Fig. 5B).

Identification and validation of hub genes. Genes with $|\mathrm{MM}+\mathrm{GS}|=5 \%$ in the black and turquoise modules were selected as candidate prognostic genes, and all other genes were removed. A PPI network of all genes in the black and turquoise modules was constructed using Cytoscape. The network comprised 222 nodes and 1,416 edges according to the STRING database (Fig. 6). Among those, C-X-C motif chemokine receptor 4 (CXCR4), interleukin 7 receptor (IL7R) and phosphatidylinositol-4,5-bisphosphate 3-kinase

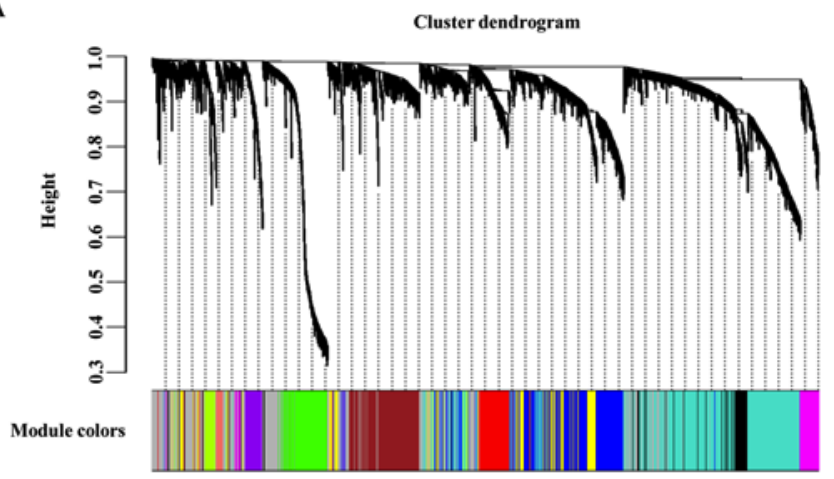

B

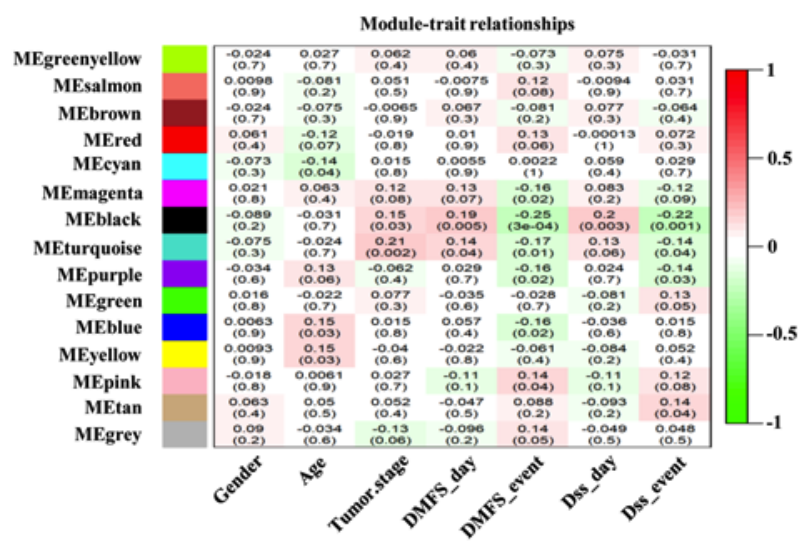

Figure 3. Identification of modules associated with the progression of melanoma. (A) Dendrogram of all differentially expressed genes clustered based on a dissimilarity measure. (B) Heatmap of the association between module eigengenes and the progression of melanoma. DMFS, distant metastasis-free survival; Dss, disease-specific death survival; ME, module eigengene. The red color of each box represents the positive association between the module and trait whereas the green color of each box represents the negative associations. The association of the module and trait is calculated to be between -1 and 1.

catalytic subunit $\gamma$ (PIK3CG) were positively associated with overall survival (Fig. 7D-F). Based on TCGA data, the expression levels of CXCR4, IL7R and PI3KG were upregulated in primary tumors compared with metastatic tumors (Fig. 7A-C). In addition, the ROC curves indicated that CXCR4, IL7R and PI3KG exhibited excellent efficacy for diagnosing primary and metastatic tumor tissues (Fig. 7G-I).

Screening candidates for treatment. Using genes which were identified as hub nodes in the PPI network (degree $>30$ ) associated with prognosis $(\mathrm{P}<0.05)$ and metastasis $(\mathrm{AUC}>0.7)$ as potential targets in the drug-gene interaction analysis (Fig. 8), the top 15 drugs ranked by the proximity of genes and drugs were screened as possible treatments for melanoma. The screened drugs could be divided into several major categories, including tyrosine kinase inhibitors (TKIs), vascular endothelial growth factor receptor (VEGFR) inhibitors, estrogen receptor modulators, proteasome inhibitors, Burton's tyrosine kinase (BTK) inhibitors and Raf kinase inhibitors. The top 15 drugs are: Ponatinib, nintedanib, tamoxifen, framycetin, regorafenib, dasatinib, sunitinib, bosutinib, benzylpenicilloyl polylysine, ibrutinib, pazopanib, methyl aminolevulinate, bortezomib, sorafenib, lenvatinib. 
A

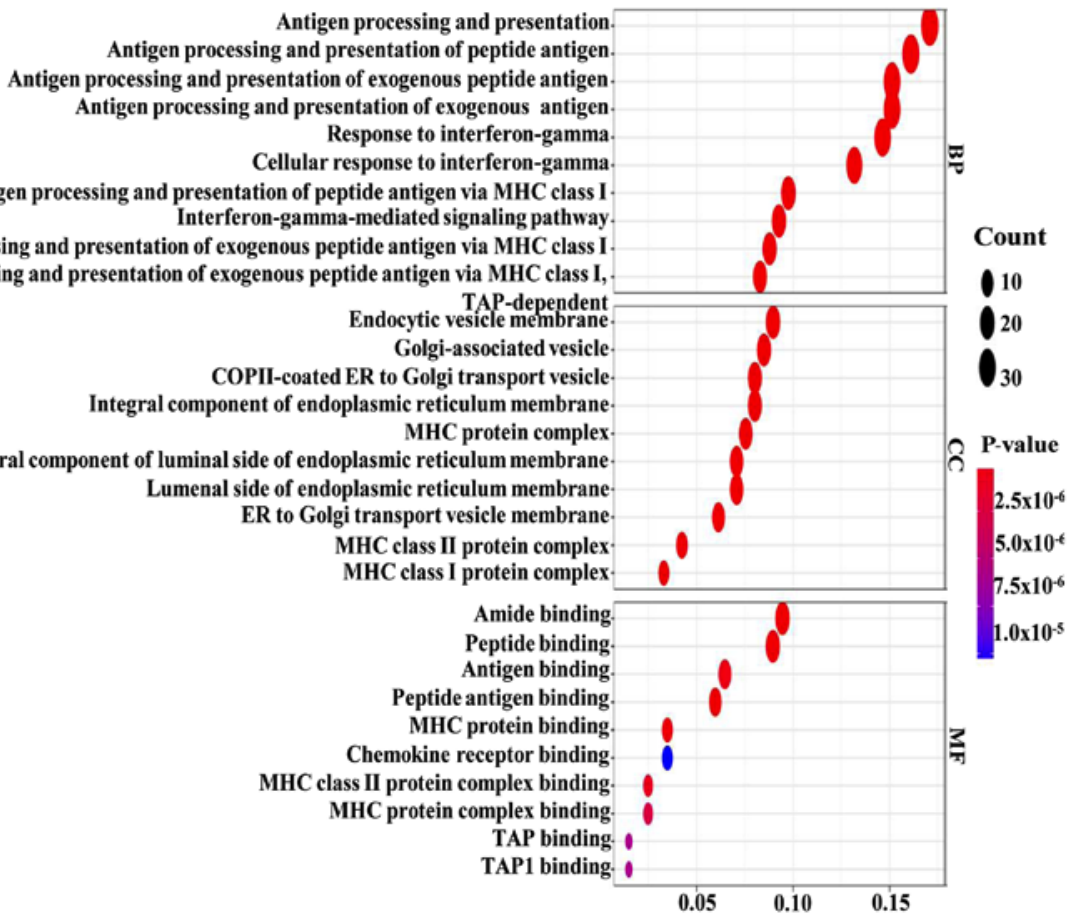

B

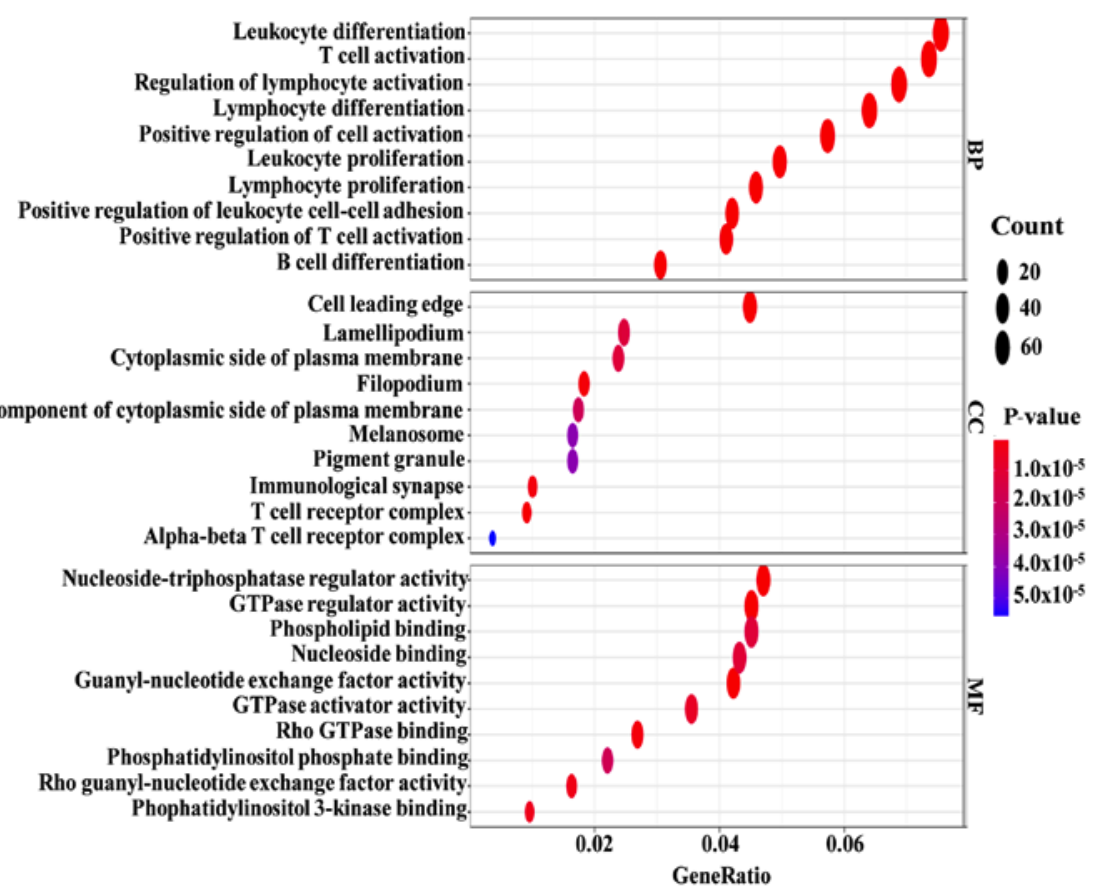

Figure 4. Gene Ontology analysis of the genes in the black and turquoise modules. (A) Gene Ontology analysis of the genes in the black module. (B) Gene Ontology analysis of the genes in the turquoise module. BP, biological process; $\mathrm{CC}$, cellular component; MF, molecular function.

\section{Discussion}

Among skin tumors, melanoma is the most malignant (22). High recurrence and metastasis rates affect the efficacy of melanoma treatment (23). The effects of conventional chemotherapy, immunotherapy and targeted therapy remain limited. Thus, identifying novel molecular targets and exploring therapeutic drugs for melanoma is important. In the present study, the GEO database was used to obtain genetic and clinical information from patients with melanoma, construct a co-expression network, select the most significant module and identify three hub genes: CXCR4, IL7R and PIK3CG. TCGA, which was used for further verification, revealed that three aforementioned specific molecules: CXCR4, IL7R and PIK3CG identified in melanoma tissues were associated with prognosis and metastasis. In addition, the top 15 drugs ranked by the proximity of genes and drugs were screened using a network screening method, and a drug-gene network was constructed.

CXCR4, which is a receptor of C-X-C motif chemokine 12 (CXCL12), is located on the surface of $>23$ human tumors, for example breast cancer, ovarian cancer, glioma, pancreatic 
A

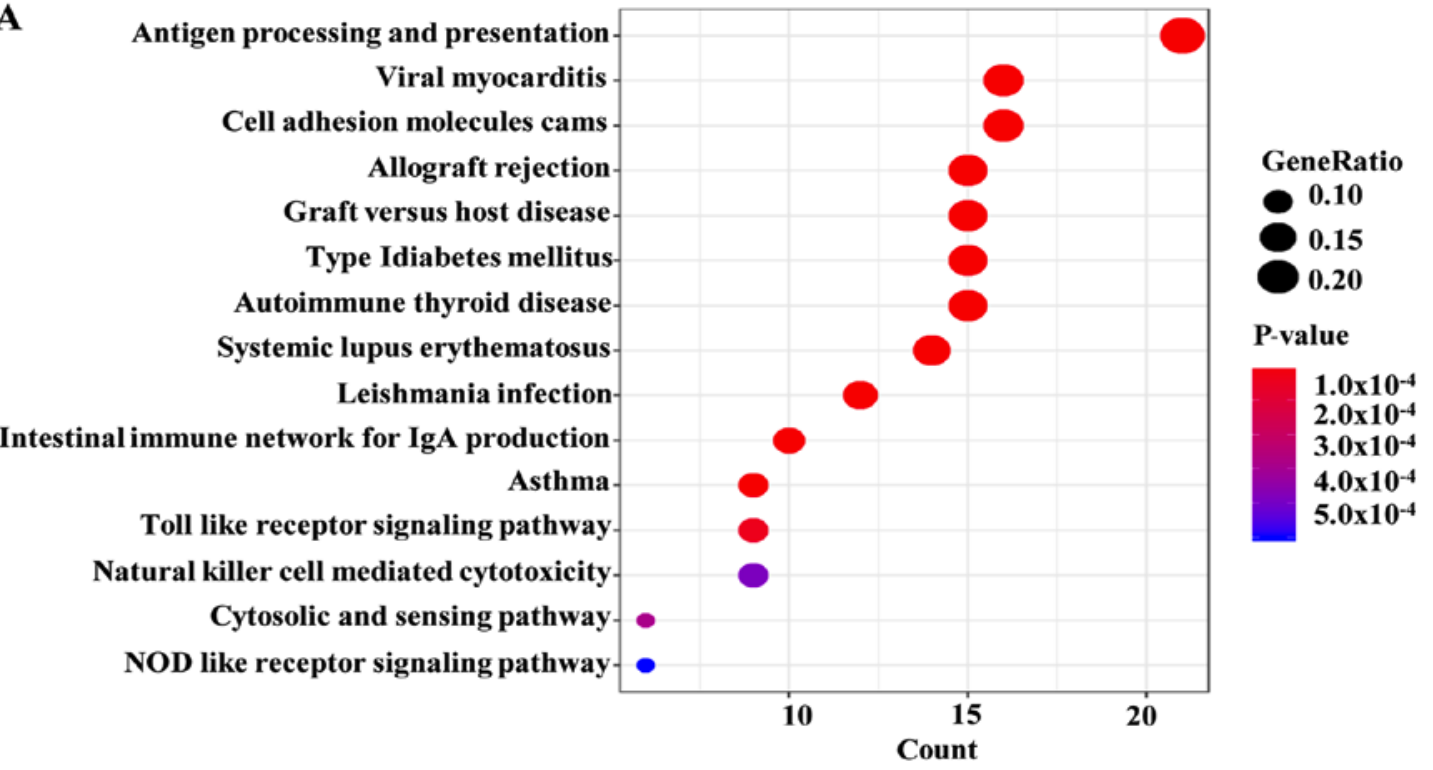

B

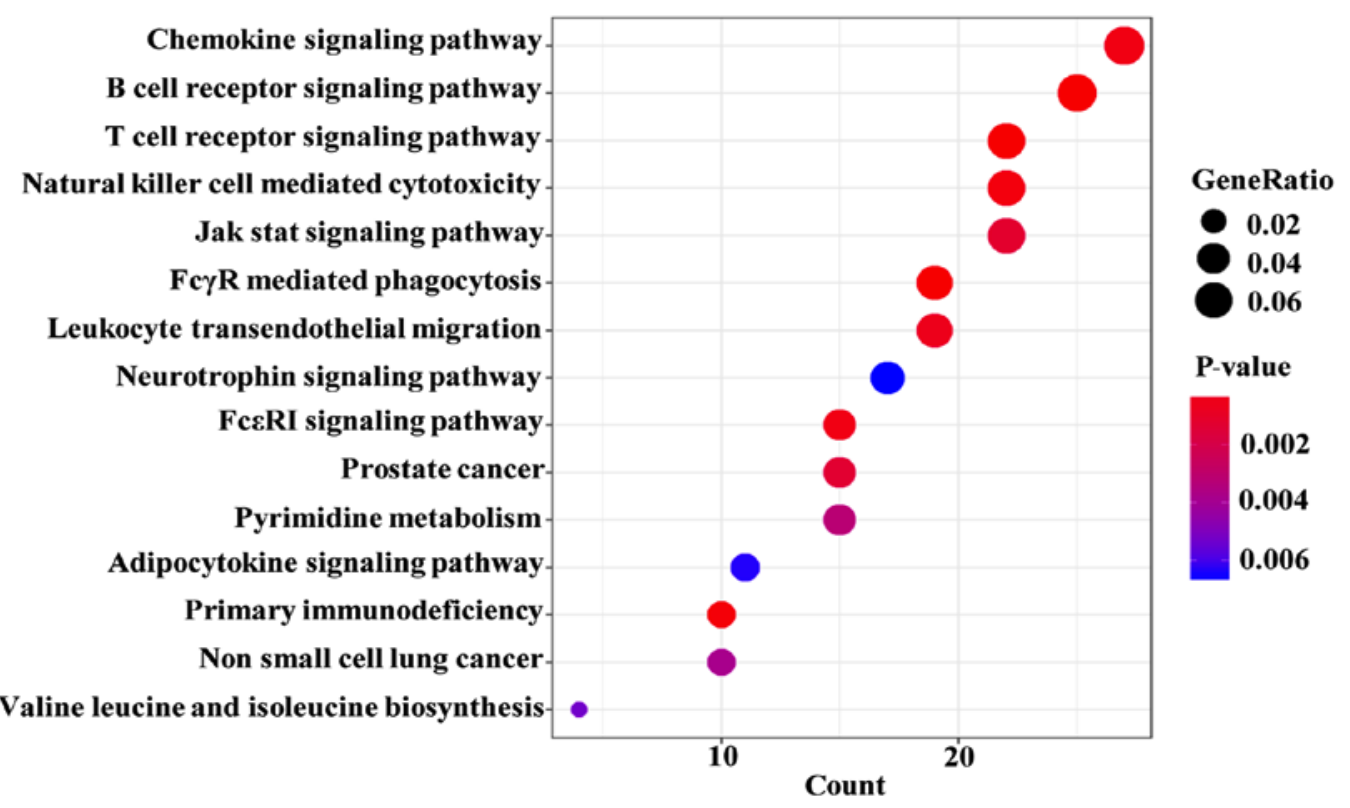

Figure 5. KEGG analysis of the genes in the black and turquoise modules. (A) KEGG analysis of the genes in the black module. (B) KEGG analysis of the genes in the turquoise module. KEGG, Kyoto Encyclopedia of Genes and Genomes.

cancer and prostate cancer (24). CXCL12 binds to CXCR4, which activates several extra- and intracellular signaling pathways, including the nuclear factor $\kappa \mathrm{B}, \mathrm{Ca}^{2+}$-dependent protein tyrosine kinase $2 \beta$, PI3K-Akt and mitogen-activated protein kinase signaling pathways (25). In various types of cancer, such as oral (26), esophageal (27), gastric, colon, liver, pancreatic, thyroid and ovarian cancer (28), and leukemia (29), CXCR4 expression is strongly associated with chemotaxis, invasion, angiogenesis and cell proliferation, all of which are involved in tumorigenesis and cancer. However, the results of the present study indicated that, compared with primary tumors, CXCR4 is downregulated in metastatic tumors, and is therefore associated with good prognosis in patients with melanoma. Mitchell et al (30) demonstrated that most of melanoma cases with mitosis, ulceration and regression were CXCR4-negative. Patients with American Joint Committee on Cancer (AJCC) stage (31) I and II melanoma exhibit higher expression of
CXCR4 compared with those with AJCC stages III and IV, and a proportion of patients with AJCC stage III-IV melanoma are CXCR4-negative (30). Therefore, the role of CXCR4 as a biomarker warrants further investigation.

IL7R, which is expressed in immune cells, is crucial for the survival, development and homeostasis of the immune system (32). IL-7R $\alpha$ activates Janus kinases 1 and 3, promoting the function of signal transducer and activator of transcription 5 , which leads to the modulation of gene expression, as well as the activation of anti-apoptotic and pro-survival signaling pathways (33). Thus, IL7R is classified as an oncogene associated with several tumors, including esophageal and prostate cancer (34). However, a bioinformatics study has demonstrated that patients with colon cancer lacking IL7R (two cases of mortality out of three cases) had a median survival time of 34 months compared with patients with normal IL7R status, whose survival time was 45 months (35). Studies on 


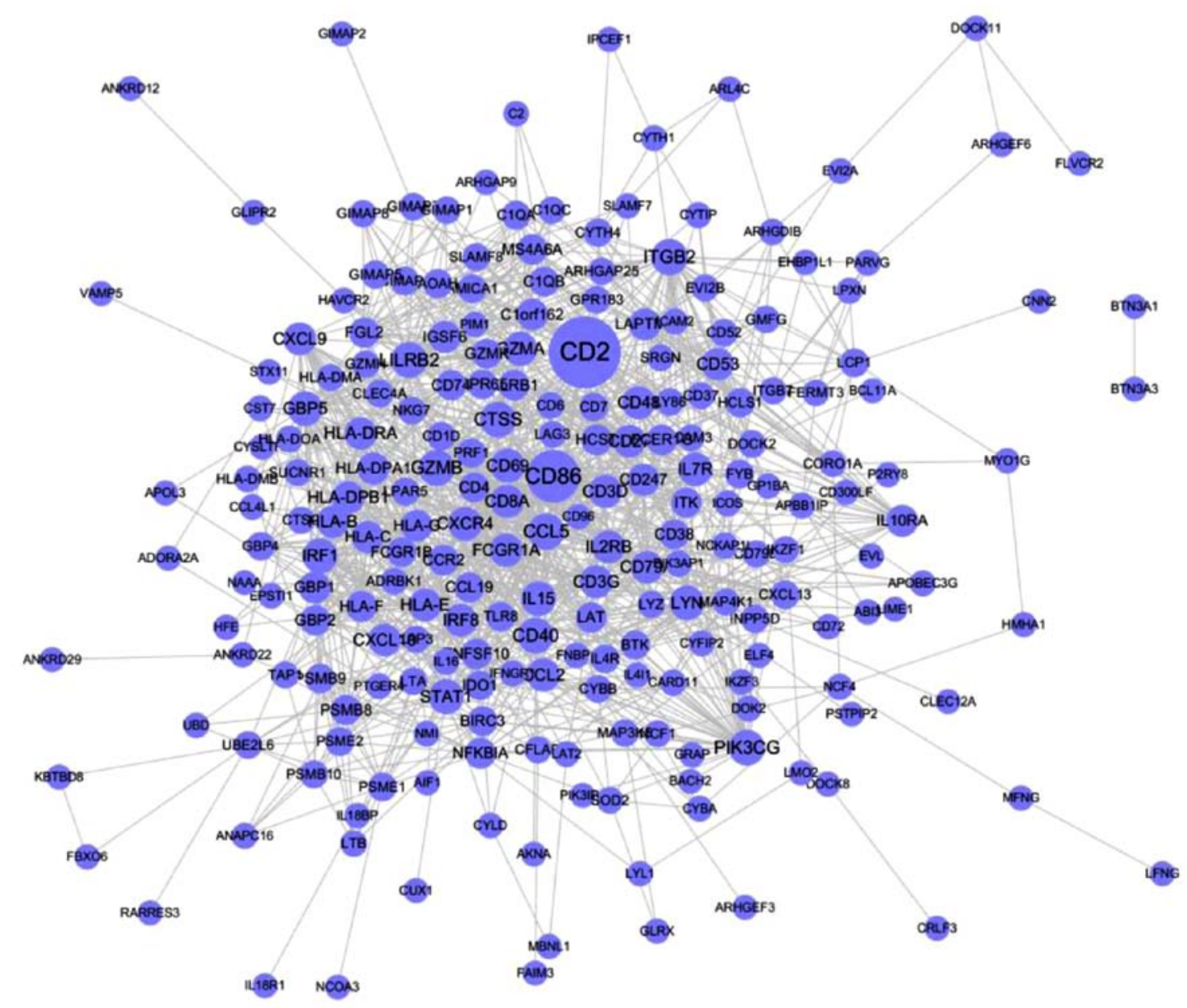

Figure 6. Protein-protein interaction network of genes in the black and turquoise modules. The size of the circle represents the degree of the node; lines indicate interactions between genes.

the association between IL7R and melanoma, as well as the association between IL7R and metastasis, are lacking.

The PI3K signaling pathway modulates various biological processes, including cell proliferation, survival, motility, death and metabolism (36). Aberrations in these processes are pivotal for the pathogenesis of cancer. Based on structural differences, PI3K can be divided into several subunits, including PIK3CA, PIK3CB, PIK3CD and PIK3CG (37). A previous study has revealed that PIK3CG is expressed at undetectable levels in glioblastoma cells, and that blocking this specific subunit does not cause cytotoxicity (38). Another study has demonstrated that PIK3CG is downregulated in colorectal cancer, whereas 12 other genes in the PI3K-AKT signaling pathway are upregulated (39). However, a bioinformatics-based study reported that PIK3CG is significantly associated with melanoma metastasis to regional lymph nodes, which contradicted the results of the present study, suggesting that further investigation may be required to clarify the role of PIK3CG in the metastasis of melanoma (40).

In the present study, the GEO database, which comprised 214 melanoma samples, and TCGA database, which included 417 patients, were selected to verify the roles of the identified genes. Double validation and a large number of samples contributed to the reliability of the candidate genes. However, a limitation of the present study was a lack of clinical or experimental validation. Further study is required to verify the role of CXCR4, IL7R and PI3K3CG in melanoma.

The analysis of the association between genes and FDA-approved drugs demonstrated that the top 15 drugs were TKIs, VEGFR inhibitors, estrogen receptor modulators, proteasome inhibitors, Bcr-Abl kinase inhibitors, BTK inhibitors, Raf kinase inhibitors, framycetin, benzylpenicilloyl polylysine and methyl aminolevulinate. TKIs that function by blocking the Bcr-Abl tyrosine-kinase included dasatinib, ponatinib and bosutinib, which are used to treat chronic myelogenous leukemia and acute lymphocytic leukemia (41). Other drugs, including nintedanib, regorafen, sunitinib, pazopanib, sorafenib and lenvatinib inhibit several receptor tyrosine kinases, including platelet-derived growth factors, VEGFR, fibroblast growth factor receptors and Raf family kinases, which inhibit tumor angiogenesis and tumor cell proliferation (42). Ibrutinib, a BTK inhibitor, is used to treat chronic lymphocytic leukemia (43). Tamoxifen, a selective estrogen receptor modulator, is used for the treatment and prevention of estrogen receptor-positive breast cancer (44). Bortezomib was the first therapeutic proteasome inhibitor to be tested in humans; it serves a role in cell cycle arrest and apoptosis, and is approved in the United States for the treatment of relapsed multiple myeloma and mantle cell lymphoma (45). Framycetin, which is an antibiotic, is used to treat leg ulcers and other conditions associated with 

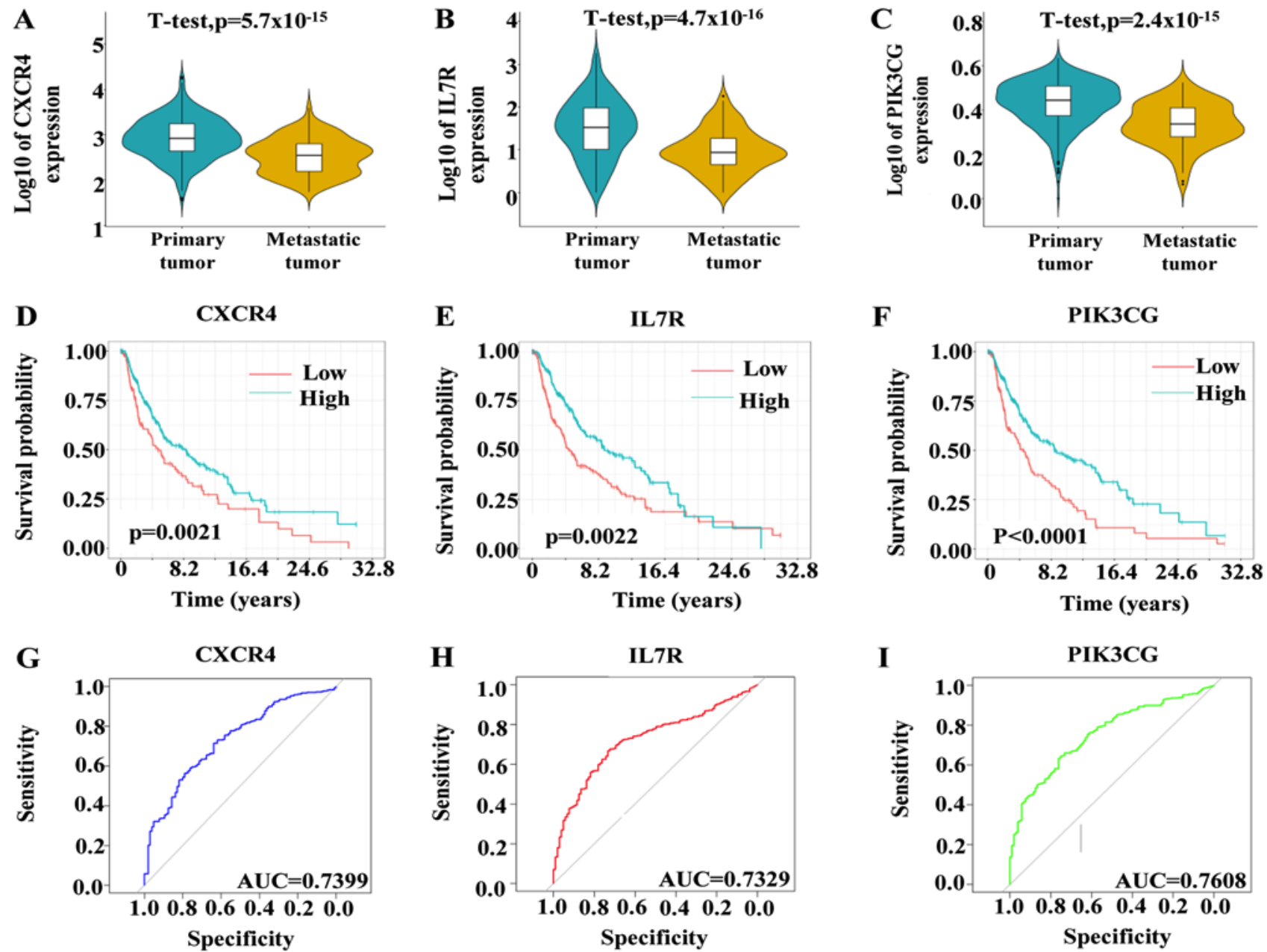

Figure 7. Validation of the expression of hub genes in primary and metastatic melanoma using TCGA database. Expression levels of (A) CXCR4, (B) IL7R and (C) PIK3CG in primary and metastatic melanoma. (D-F) Survival analysis of the hub genes in TCGA dataset for (D) CXCR4, (E) IL7R and (F) PIK3CG. Red lines represent low expression of the hub genes; blue lines represent high expression. (G-I) Receiver operating characteristic curves and AUC statistics were calculated to evaluate the capacity of distinguishing primary and metastatic melanoma of (G) CXCR4, (H) IL7R and (I) PIK3CG. TGCA, The Cancer Genome Atlas; CXCR4, C-X-C motif chemokine receptor 4; IL7R, interleukin 7 receptor; PIK3CG, phosphatidylinositol-4,5-bisphosphate 3-kinase catalytic subunit $\gamma$; AUC, area under the curve.

wound healing (46). Benzylpenicilloyl polylysine is used as a skin-testing reagent for individuals with a history of penicillin allergy (47). Methyl aminolevulinate, which is metabolized into phototoxic compounds, such as protopophyrin IX, may represent a candidate for photodynamic therapy, as it can induce oxidative damage to the cell (48).

Angiogenesis is a hallmark of several types of tumor, including melanoma. The process of angiogenesis is crucial for tumor development and metastasis (49). VEGF is one of the most important cytokines responsible for tumor-mediated angiogenesis (50). VEGF is strongly expressed in melanoma and serves a critical role in the progression of the disease (51). In a phase II study of sunitinib in patients with advanced melanoma, 4/31 (13\%) patients exhibited a partial response and $8(26 \%)$ had stable disease (52). Pazopanib, a VEGF and platelet-derived growth factor inhibitor, has been used in combination with paclitaxel in a phase II study of patients with metastatic melanoma; the 6 -month progression-free survival rate was $68 \%$, and the 1 -year overall survival rate was $48 \%$ (53). The objective response rate was $37 \%$, comprising one complete and 20 partial responses (54). A phase Ib study using lenvatinib (E7080) in combination with temozolomide for the treatment of advanced melanoma indicated an overall objective response rate of $18.8 \%$ (six patients), comprising all partial responses (55). SRC proto-oncogene, non-receptor tyrosine kinase (SRC) is a promising target in the treatment of solid types of cancer, including human melanoma; bosutinib, a SRC inhibitor, which induces cell death via lysosomal membrane permeabilization in melanoma cells, is a promising therapeutic agent for melanoma treatment (56). SRC inhibitor Dasatinib specifically inhibits p53 phosphorylation in melanoma; however, a comprehensive validation is required (57). Ibrutinib, a BTK inhibitor, has been used to treat chronic lymphocytic leukemia/small lymphocytic lymphoma and subsequent melanoma that occurs following leukemia (58). Sorafenib, a Raf inhibitor, is a first-line therapeutic agent used in advanced melanoma (phase I and open-label phase II) trials with an overall response rate of $12 \%$ with one complete response and nine partial responses (59). Bortezomib administration reduces the levels of proangiogenic cytokines in plasma (60). A clinical 


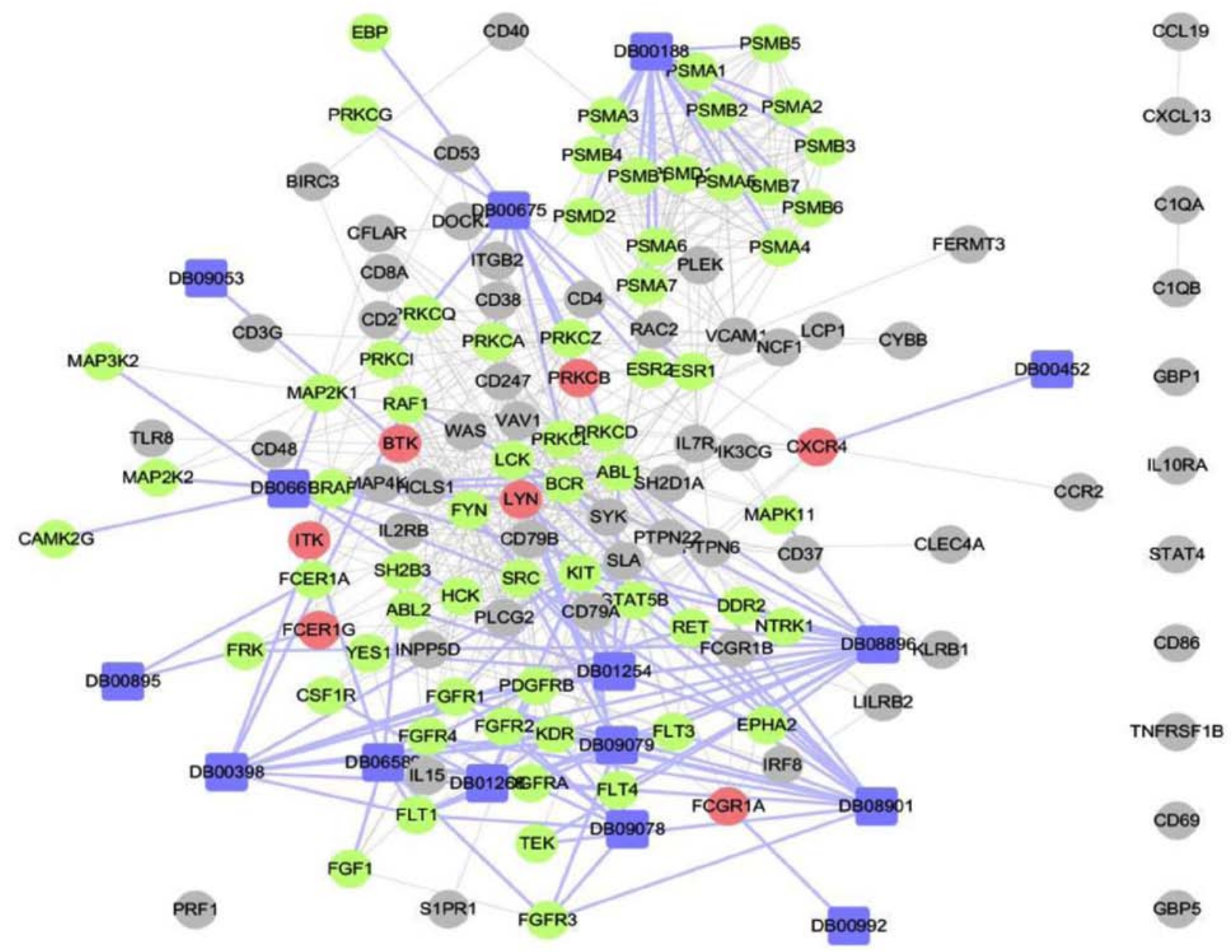

Figure 8. Drug-gene interaction network. Network analysis of hub genes and their target drug networks in melanoma. Purple squares represent predicted drugs that may treat melanoma, pink dots are drug targets, green dots are genes associated with tumor development that are also drug targets, and gray dots are genes associated with tumor development that are not targets of drugs. A purple line indicates an interaction between a drug and a target, and a gray line indicates an interaction between genes.

trial has indicated that tamoxifen therapy is not effective for treating melanoma, and that the mode of action of antiestrogens in melanoma is unclear (61). To the best of our knowledge, ponatinib, nintedanib, regorafen, framycetin, benzylpenicilloyl polylysine and methyl aminolevulinate have not been used to treat melanoma.

The present study used WGCNA to construct a gene co-expression network in order to determine the associations between genes and modules and to explore the association between the gene modules and clinical characteristics. Two significant modules (black and turquoise modules) shown in Fig. 3, were identified to be associated with the progression of melanoma. GO and KEGG pathway analyses demonstrated that this module was mostly involved in functions associated with antigen presentation. In addition, three hub genes, CXCR4, IL7R and PI3K3CG, were identified and demonstrated to be associated with the progression and prognosis of melanoma. Analysis of the interaction between genes and drug targets of the top 15 drugs for melanoma enabled the construction of a network of drug-gene interactions. Ponatinib, regorafen, nintedanib, framycetin, benzyl penicilloyl polylysine and methyl aminolevulinate, which were among the 15 drugs not currently used to treat melanoma, may be potential novel therapeutic drugs for this disease.

\section{Acknowledgements}

Not applicable.

\section{Funding}

No funding was received.

\section{Availability of data and materials}

The datasets used and/or analyzed during the current study are available from the corresponding author on reasonable request.

\section{Authors' contributions}

JYG conceived and designed the study. XYD, QW, SMZ and JHY acquired and interpreted the data. LW, CYW, YYX, XY, TFX and YYP conducted the data analyses. LW, CYW and YYX wrote the manuscript. JYG revised the manuscript. All authors read and approved the final manuscript and agreed to be accountable for all aspects of the research in ensuring that the accuracy or integrity of any part of the work are appropriately investigated and resolved. 


\section{Ethics approval and consent to participate}

Not applicable.

\section{Patient consent for publication}

Not applicable.

\section{Competing interests}

The authors declare that they have no competing interests.

\section{References}

1. Colebatch AJ and Scolyer RA: Trajectories of premalignancy during the journey from melanocyte to melanoma. Pathology 50 16-23, 2018.

2. Slominski A, Tobin DJ, Shibahara S and Wortsman J: Melanin pigmentation in mammalian skin and its hormonal regulation. Physiol Rev 84: 1155-1228, 2004.

3. Slominski A, Zmijewski MA and Pawelek J: L-tyrosine and L-dihydroxyphenylalanine as hormone-like regulators of melanocyte functions. Pigment Cell Melanoma Res 25: 14-27, 2012.

4. Slominski A, Kim TK, Brozyna AA, Janjetovic Z, Brooks DL, Schwab LP, Skobowiat C, Jóźwicki W and Seagroves TN: The role of melanogenesis in regulation of melanoma behavior: Melanogenesis leads to stimulation of HIF-1 $\alpha$ expression and HIF-dependent attendant pathways. Arch Biochem Biophys 563: 79-93, 2014.

5. Slominski RM, Zmijewski MA and Slominski AT: The role of melanin pigment in melanoma. Exp Dermatol 24: 258-259, 2015

6. Hyams DM, Cook RW and Buzaid AC: Identification of risk in cutaneous melanoma patients: Prognostic and predictive markers. J Surg Oncol 119: 175-186, 2019.

7. Ugurel S, Rohmel J, Ascierto PA,Flaherty KT, Grob JJ, Hauschild A, Larkin J, Long GV, Lorigan P, McArthur GA, et al: Survival of patients with advanced metastatic melanoma: The impact of novel therapies-update 2017. Eur J Cancer 83: 247-257, 2017.

8. Read J, Wadt KA and Hayward NK: Melanoma genetics. J Med Genet 53: 1-14, 2016.

9. Koelblinger P, Dornbierer J and Dummer R: A review of binimetinib for the treatment of mutant cutaneous melanoma. Future Oncol 13: 1755-1766, 2017

10. Silva IP and Long GV: Systemic therapy in advanced melanoma: Integrating targeted therapy and immunotherapy into clinical practice. Curr Opin Oncol 29: 484-492, 2017.

11. da Silveira Nogueira Lima JP, Georgieva M, Haaland B and de Lima Lopes G: A systematic review and network meta-analysis of immunotherapy and targeted therapy for advanced melanoma. Cancer Med 6: 1143-1153, 2017.

12. Appenzeller S, Gesierich A, Thiem A, Hufnagel A, Jessen C, Kneitz H, Regensburger M, Schmidt C, Zirkenbach V, Bischler T, et al: The identification of patient-specific mutations reveals dual pathway activation in most patients with melanoma and activated receptor tyrosine kinases in BRAF/NRAS wild-type melanomas. Cancer 125: 586-600, 2019.

13. Winder M and Virós A: Mechanisms of drug resistance in melanoma. Handb Exp Pharmacol 249: 91-108, 2018.

14. Slominski AT and Carlson JA: Melanoma resistance: A bright future for academicians and a challenge for patient advocates. Mayo Clin Proc 89: 429-433, 2014.

15. Cirenajwis H, Ekedahl H, Lauss M, Harbst K, Carneiro A, Enoksson J, Rosengren F, Werner-Hartman L, Törngren T, Kvist A, et al: Molecular stratification of metastatic melanoma using gene expression profiling: Prediction of survival outcome and benefit from molecular targeted therapy. Oncotarget 6 : 12297-12309, 2015.

16. Tang J, Kong D, Cui Q, Wang K, Zhang D, Gong Y and Wu G: Prognostic genes of breast cancer identified by gene Co-expression network analysis. Front Oncol 8: 374, 2018.

17. Rahmani B, Zimmermann MT, Grill DE, Kennedy RB Oberg AL, White BC, Poland GA and McKinney BA: Recursive indirect-paths modularity (RIP-M) for detecting community structure in RNA-Seq Co-expression networks. Front Genet 7: $80,2016$.
18. Gene Ontology Consortium: Gene ontology consortium: Going forward. Nucleic Acids Res 43 (Database Issue): D1049-D1056, 2015.

19. Kanehisa M, Sato Y, Kawashima M, Furumichi M and Tanabe M: KEGG as a reference resource for gene and protein annotation. Nucleic Acids Res 44: D457-D462, 2016.

20. Su G, Morris JH, Demchak B and Bader GD: Biological network exploration with Cytoscape 3. Curr Protoc Bioinformatics 47: 8.13.1-24, 2014.

21. Cheng F, Desai RJ, Handy DE, Wang R, Schneeweiss S, Barabási AL and Loscalzo J: Network-based approach to prediction and population-based validation of in silico drug repurposing. Nat Commun 9: 2691, 2018.

22. Rajabi P, Bagheri M and Hani M: Expression of estrogen receptor alpha in malignant melanoma. Adv Biomed Res 6: 14, 2017.

23. Testori A, Ribero S and Bataille V: Diagnosis and treatment of in-transit melanoma metastases. Eur J Surg Oncol 43: 544-560, 2017.

24. Balkwill F: Cancer and the chemokine network. Nat Rev Cancer 4: 540-550, 2004.

25. Nazari A,KhorramdelazadH and Hassanshahi G: Biological/pathological functions of the CXCL12/CXCR4/CXCR7 axes in the pathogenesis of bladder cancer. Int J Clin Oncol 22: 991-1000, 2017.

26. Ishikawa T, Nakashiro K, Hara S, Klosek SK, Li C, Shintani S and Hamakawa H: CXCR4 expression is associated with lymph-node metastasis of oral squamous cell carcinoma. Int J Oncol 28: 61-66, 2006.

27. Wu J, Wu X, Liang W, Chen C, Zheng L and An $\mathrm{H}$ Clinicopathological and prognostic significance of chemokine receptor CXCR4 overexpression in patients with esophageal cancer: A meta-analysis. Tumour Biol 35: 3709-3715, 2014.

28. Kodama J, Hasengaowa, Kusumoto T, Seki N, Matsuo T, Ojima Y, Nakamura K, Hongo A and Hiramatsu Y: Association of CXCR4 and CCR7 chemokine receptor expression and lymph node metastasis in human cervical cancer. Ann Oncol 18: 70-76, 2007.

29. Konoplev S, Rassidakis GZ, Estey E, Kantarjian H, Liakou CI, Huang X, Xiao L, Andreeff M, Konopleva M and Medeiros LJ: Overexpression of CXCR4 predicts adverse overall and event-free survival in patients with unmutated FLT3 acute myeloid leukemia with normal karyotype. Cancer 109: 1152-1156, 2007.

30. Mitchell B, Leone D, Feller K, Menon S, Bondzie P, Yang S, Park HY and Mahalingam M: Protein expression of the chemokine receptor CXCR4 and its ligand CXCL12 in primary cutaneous melanoma-biomarkers of potential utility? Hum Pathol 45: 2094-2100, 2014.

31. Balch CM, Gershenwald JE, Soong SJ, Thompson JF, Atkins MB, Byrd DR, Buzaid AC, Cochran AJ, Coit DG, Ding S, et al: Final version of 2009 AJCC melanoma staging and classification. J Clin Oncol 27: 6199-6206, 2009.

32. Leung GA, Cool T, Valencia CH, Worthington A, Beaudin AE and Forsberg EC: The lymphoid-associated interleukin 7 receptor (IL7R) regulates tissue-resident macrophage development. Development 146: pii: dev176180, 2019.

33. Vitiello G, Losi GR, Amarante MK, Ceribelli JR, Carmelo E and Watanabe M: Interleukin 7 receptor alpha Thr244Ile genetic polymorphism is associated with susceptibility and prognostic markers in breast cancer subgroups. Cytokine 103: 121-126, 2018.

34. Kim MJ, Choi SK, Hong SH, Eun JW, Nam SW, Han JW and You JS: Oncogenic IL7R is downregulated by histone deacetylase inhibitor in esophageal squamous cell carcinoma via modulation of acetylated FOXO1. Int J Oncol 53: 395-403, 2018.

35. Oliveira DM, Santamaria G, Laudanna C, Migliozzi S, Zoppoli P, Quist M, Grasso C, Mignogna C, Elia L, Faniello MC, et al: Identification of copy number alterations in colon cancer from analysis of amplicon-based next generation sequencing data. Oncotarget 9: 20409-20425, 2018.

36. Vivanco I and Sawyers CL: The phosphatidylinositol 3-Kinase AKT pathway in human cancer. Nat Rev Cancer 2: 489-501, 2002.

37. Thorpe LM, Yuzugullu H and Zhao JJ: PI3K in cancer: Divergent roles of isoforms, modes of activation and therapeutic targeting. Nat Rev Cancer 15: 7-24, 2015.

38. Pridham KJ, Varghese RT and Sheng Z: The role of Class IA phosphatidylinositol-4,5-bisphosphate 3-kinase catalytic subunits in glioblastoma. Front Oncol 7: 312, 2017.

39. Semba S, Itoh N, Ito M, Youssef EM, Harada M, Moriya T, Kimura W and Yamakawa M: Down-regulation of PIK3CG, a catalytic subunit of phosphatidylinositol 3-OH kinase, by $\mathrm{CpG}$ hypermethylation in human colorectal carcinoma. Clin Cancer Res 8: 3824-3831, 2002 . 
40. Gorlov I, Orlow I, Ringelberg C, Hernando E, Ernstoff MS, Cheng C, Her S, Parker JS, Thompson CL, Gerstenblith MR, et al: Identification of gene expression levels in primary melanoma associated with clinically meaningful characteristics. Melanoma Res 28: 380-389, 2018.

41. Abou DI, Jabbour E, Short NJ and Ravandi F: Treatment of philadelphia chromosome-positive acute lymphoblastic leukemia. Curr Treat Options Oncol 20: 4, 2019.

42. Zhao Y and Adjei AA: Targeting angiogenesis in cancer therapy: Moving beyond vascular endothelial growth factor. Oncologist 20: 660-673, 2015.

43. Deeks ED: Ibrutinib: A review in chronic lymphocytic leukaemia. Drugs 77: 225-236, 2017.

44. Shagufta and Ahmad I: Tamoxifen a pioneering drug: An update on the therapeutic potential of tamoxifen derivatives. Eur J Med Chem 143: 515-531, 2018.

45. Guerrero-Garcia TA, Mogollon RJ and Castillo JJ: Bortezomib in plasmablastic lymphoma: A glimpse of hope for a hard-to-treat disease. Leuk Res 62: 12-16, 2017.

46. Rai R, Shenoy MM, Viswanath V, Sarma N, Majid I and Dogra S: Contact sensitivity in patients with venous leg ulcer: A multi-centric Indian study. Int Wound J 15: 618-622, 2018.

47. Vemuri P, Harris KE, Suh LA and Grammer LC: Preparation of benzylpenicilloyl-polylysine: A preliminary study. Allergy Asthma Proc 25: 165-168, 2004.

48. Yazdanyar S, Zarchi K and Jemec GBE: Pain during topical photodynamic therapy-comparing methyl aminolevulinate $\left(\right.$ Metvix ${ }^{\circledR}$ ) to aminolaevulinic acid $\left(\right.$ Ameluz $\left.^{\circledR}\right)$; an intra-individual clinical study. Photodiagnosis Photodyn Ther 20: 6-9, 2017.

49. Jour G, Ivan D and Aung PP: Angiogenesis in melanoma: An update with a focus on current targeted therapies. J Clin Pathol 69: 472-483, 2016.

50. Ribatti D: Tumor refractoriness to anti-VEGF therapy. Oncotarget 7: 46668-46677, 2016.

51. Ott PA, Hodi FS and Buchbinder EI: Inhibition of immune checkpoints and vascular endothelial growth factor as combination therapy for metastatic melanoma: An overview of rationale, preclinical evidence, and initial clinical data. Front Oncol 5: 202, 2015.

52. Decoster L, Vande BI, Neyns B, Majois F, Baurain JF, Rottey S Rorive A, Anckaert E, De Mey J, De Brakeleer S and De Grève J: Biomarker analysis in a Phase II study of sunitinib in patients with advanced melanoma. Anticancer Res 35: 6893-6899, 2015.
53. Urbonas V, Schadendorf D, Zimmer L, Danson S, Marshall E, Corrie P, Wheater M, Plummer E, Mauch C, Scudder C, et al: Paclitaxel with or without trametinib or pazopanib in advanced wild-type BRAF melanoma (PACMEL): A multicentre, open-label, randomised, controlled phase II trial. Ann Oncol 30: 317-324, 2019.

54. Fruehauf JP, El-Masry M, Osann K, Parmakhtiar B, Yamamoto M and Jakowatz JG: Phase II study of pazopanib in combination with paclitaxel in patients with metastatic melanoma. Cancer Chemother Pharmacol 82: 353-360, 2018.

55. Hong DS, Kurzrock R, Falchook GS, Andresen C, Kwak J, Ren M, Xu L, George GC, Kim KB, Nguyen LM, et al: Phase 1b study of lenvatinib (E7080) in combination with temozolomide for treatment of advanced melanoma. Oncotarget 6: 43127-43134, 2015.

56. Noguchi S, Shibutani S, Fukushima K, Mori T, Igase M and Mizuno T: Bosutinib, an SRC inhibitor, induces caspase-independent cell death associated with permeabilization of lysosomal membranes in melanoma cells. Vet Comp Oncol 16: 69-76, 2018.

57. Skoko J, Rožanc J, Charles EM, Alexopoulos LG and Rehm M: Post-treatment de-phosphorylation of p53 correlates with dasatinib responsiveness in malignant melanoma. BMC Cell Biol 19: 28, 2018.

58. Archibald WJ, Meacham PJ, Williams AM, Baran AM, Victor AI, Barr PM, Sahasrahbudhe DM and Zent CS: Management of melanoma in patients with chronic lymphocytic leukemia. Leuk Res 71: 43-46, 2018

59. Eisen T, Marais R, Affolter A, Lorigan P, Robert C, Corrie P, Ottensmeier C, Chevreau C, Chao D, Nathan PD, et al: Sorafenib and dacarbazine as first-line therapy for advanced melanoma: Phase I and open-label phase II studies. Br J Cancer 105: 353-359, 2011.

60. Rossi UA, Finocchiaro L and Glikin GC: Bortezomib enhances the antitumor effects of interferon- $\beta$ gene transfer on melanoma cells. Anticancer Agents Med Chem 17: 754-761, 2017.

61. Ribeiro MP, Santos AE and Custódio JB: Rethinking tamoxifen in the management of melanoma: New answers for an old question. Eur J Pharmacol 764: 372-378, 2015.

This work is licensed under a Creative Commons Attribution-NonCommercial-NoDerivatives 4.0 International (CC BY-NC-ND 4.0) License. 